\title{
DNA molecules resolved by electrical double layer force spectroscopy imaging
}

\author{
J. Sotres and A. M. Baróa) \\ Instituto de Ciencia de Materiales de Madrid, CSIC, E-28049 Madrid, Spain
}

(Received 7 July 2008; accepted 15 August 2008; published online 11 September 2008)

\begin{abstract}
This paper deals with spatially resolved force spectroscopy applied to the imaging of the electrical double layer (EDL) force. It is focused on an important point that has not been elucidated yet, the resolution that can be achieved. For this purpose, single plasmid DNA molecules have been studied with an atomic force microscope operated in the force spectroscopy imaging mode. With this approach, important quantities deduced from the force spectra can be simultaneously mapped along with the topography image. Single DNA molecules have been probed obtaining a lateral resolution in the EDL force similar to that of the topography. (C) 2008 American Institute of Physics.
\end{abstract}

[DOI: 10.1063/1.2981688]

Atomic force microscopy (AFM) has proved to be a useful technique in imaging biological molecules in liquid environment with subnanometer resolution. ${ }^{1}$ Moreover, its capability to measure interaction forces down to the piconewton range has emerged as a field itself known as force spectroscopy. ${ }^{2}$

Charged surfaces in the presence of electrolyte solutions develop an electrical double layer (EDL) force. ${ }^{3}$ This force originated from the overlapping of the diffuse ion layers around the surfaces. In AFM, tip and substrate can be approximated by a sphere interacting with a planar surface, and the EDL force is given by ${ }^{4}$

$$
F_{\mathrm{EDL}}(d)=\frac{4 \pi \sigma_{s} \sigma_{t} R \lambda_{D}}{\varepsilon} e^{-d / \lambda_{D}} \quad\left(d>\lambda_{D}\right),
$$

where $R$ is the tip radius, $d$ is the tip-sample distance, $\sigma_{t}$ and $\sigma_{s}$ are the tip and sample surface charge densities, respectively, and $\lambda_{D}$ is the characteristic decay length of $F_{\mathrm{EDL}}$, also known as the Debye length. From Eq. (1) it follows that the EDL force is related to the sample surface charge density.

A powerful method is to combine AFM with spatially resolved force spectroscopy. Force spectroscopy imaging (FSI) (Ref. 5) involves acquiring a force-distance curve at each pixel of a simultaneously acquired AFM topography image. The same idea of force curve images was reported by other authors. ${ }^{6,7}$ The interesting point about these images is that important properties of the sample, such as elasticity, adhesion, or electrostatic forces, can be laterally resolved. One of the major limitations of FSI and similar operation modes is the low lateral resolution. This has been attributed to the long acquisition time, which enhances thermal drift effects. Indeed, only a few works focus on systems in the nanometer range. ${ }^{6,8} \mathrm{~A}$ higher number deal with systems in the micrometer range, including those where EDL interaction forces have been studied. ${ }^{9-11}$ The aim of this work is to study the resolution limits of the EDL force as measured by AFM to image biological molecules. For that purpose, we have chosen a double stranded DNA molecule of $2 \mathrm{~nm}$ diameter. We show that the molecule is resolved in the EDL force.

${ }^{a)}$ Electronic mail: abaro@icmm.csic.es.
The system of study consisted in single isolated 2686bp pUC19 plasmid DNA molecules (Sigma-Aldrich, Tres Cantos, Spain) deposited on a mica surface (Electron Microscopy Sciences, Hatfield PA) coated with polylysine (PL) (Sigma-Aldrich, Tres Cantos, Spain). For sample preparation, a $10 \mu \mathrm{l}$ PL solution was pipetted on a freshly cleaved mica sheet. After incubation for $30 \mathrm{~s}$, the mica was rinsed with $10 \mathrm{ml}$ of milliQ water and dried in a nitrogen stream. Then, the PL-coated mica surface was incubated in a $5 \mu \mathrm{g} / \mathrm{ml}$ DNA solution for $1 \mathrm{~min}$ and rinsed with phosphate buffer, not allowing it to dry at any moment. Experiments were done in phosphate buffer $10 \mathrm{mM} \mathrm{pH} \mathrm{7,} \mathrm{consisting} \mathrm{of} \mathrm{a}$ $0.056 \%$ monosodium phosphate (Electron Microscopy Sciences, Hatfield PA) and $0.155 \%$ disodium phosphate (Electron Microscopy Sciences, Hatfield PA) in weight solution in milliQ water. In these conditions, PL confers a net positive charge to the surface, which is necessary to fix the negatively charged DNA molecules. The sample was probed with a silicon nitride tip (MLCT-AUMT-A, Veeco Probes, Camarillo CA) with a nominal force constant given by the manufacturer of $30 \mathrm{pN} / \mathrm{nm}$. Silicon nitride, at neutral $\mathrm{pH}$, develops a net negative surface charge. Following Eq. (1) an attractive EDL force is expected for a $\mathrm{Si}_{3} \mathrm{~N}_{4}$ tip approaching a PL surface, while the DNA molecule is expected to be repulsive.

In order to probe the EDL on top of a DNA strand, it is necessary to operate the AFM in the FSI mode. This has been done with a commercial AFM (Nanotec S. L., Tres Cantos, Spain) controlled with the WSXM software. ${ }^{12}$ In this case, FSI is implemented in a different way, as a software method running in the digital signal processor memory. It works by performing a force curve at each point of the scanned surface. At maximum tip-sample separation, the cantilever deflection corresponding to zero force is measured. Then, the sample is approached toward the tip. During this jump, the deflection signal is sampled at defined distance intervals. At the point of minimum separation, a feedback loop is closed typically for a few milliseconds. In the present application, the feedback channel has been the cantilever deflection although other signals could be used. With the feedback loop closed, the sample vertical position is adjusted so that the increment of the cantilever deflection with respect to its zeroforce value approaches a defined set value. During this time interval, the average sample vertical position is stored as the 


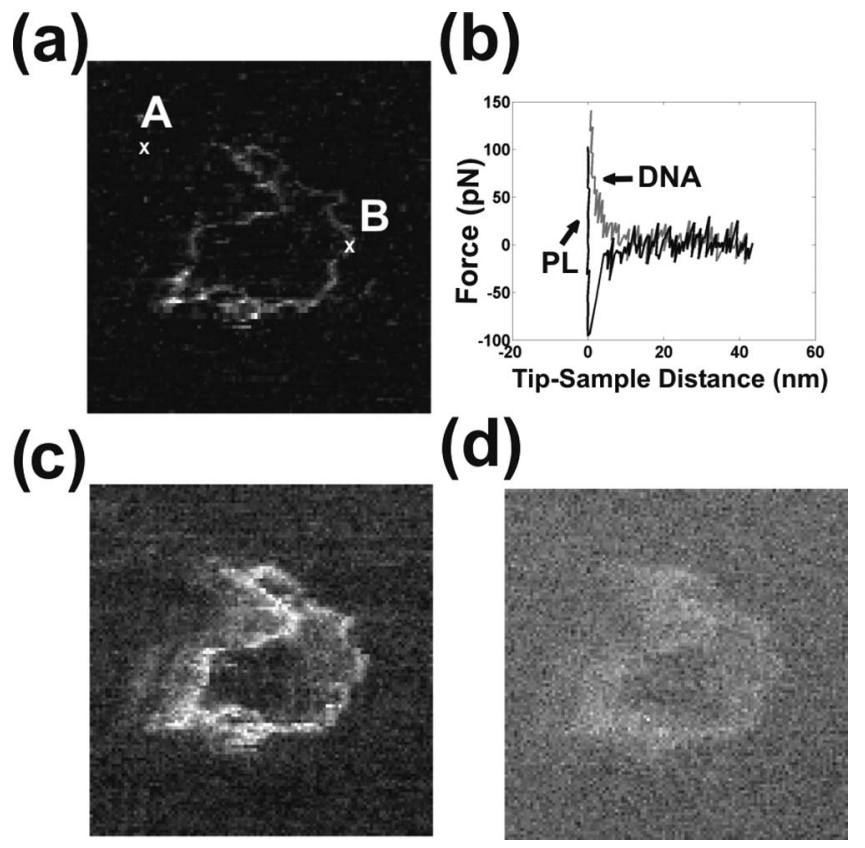

FIG. 1. (a) Topography image, from a FSI file, of a plasmid DNA molecule on a PL-coated mica surface imaged in phosphate buffer of $10 \mathrm{mM}$. The visualized surface area is $350 \times 350 \mathrm{~nm}^{2}$. Height values extend from $0 \mathrm{~nm}$ (black) to $3 \mathrm{~nm}$ (white). (b) Force scans on top of the PL-coated mica surface [black curve: point A in (a) and on top of the DNA strand; gray curve: point B in (a)]. (c) Isodistance map corresponding to the area represented in Fig. 1(a) to a distance of $3 \mathrm{~nm}$ and (d) to a distance of $6 \mathrm{~nm}$. Height color values go from -60 (black) to $+60 \mathrm{pN}$ (white).

height value for the corresponding surface point. Then, the feedback loop is opened and the sample separated from the tip for a given distance, sampling again the cantilever deflection. Finally, lateral movement is done at the point of furthest separation, avoiding lateral forces, and the cycle starts again. For each surface point, the height and the sampled deflection values during approach and retraction are transmitted to the control computer.

In Fig. 1(a) the topography of a DNA molecule, obtained by operating the AFM in the FSI mode, is shown. The FSI file consists of $128 \times 128$ array of force curves, taken at lateral intervals of $3 \mathrm{~nm}$. Force curves consist of 170 deflection values sampled at vertical intervals of $0.4 \mathrm{~nm}$. Pixel acquisition frequency was $40 \mathrm{~Hz}$ (less than $7 \mathrm{~min}$ for an image). The scanning rate was limited by the hydrodynamic drag. As for higher speeds, the drag force approaches the EDL value. The molecule shown has a height mean value of $\sim 1 \mathrm{~nm}$, smaller than the nominal of $2 \mathrm{~nm}$ as it is commonly observed in the AFM. ${ }^{13}$ The molecule mean width at half-height is $10 \mathrm{~nm}$, which can be explained by tip dilation effects. From this, we conclude that we have resolved a single plasmid DNA molecule with a resolution comparable to that obtained by other operation modes. ${ }^{14}$

Two individual representative force scans of the FSI mode are shown in Fig. 1(b). The black line (attractive) corresponds to the tip placed on top of the PL-coated mica surface [point A in Fig. 1(a)], whereas the gray line (repulsive) has been taken on top of DNA (point B). The experimentally obtained curves of force versus z-piezo displacement have been transformed into the more physically meaningful force versus tip-sample distance. ${ }^{15}$ Since tip size is bigger than DNA, when scanning the molecule the tip is probing both substrate and DNA. This induces a topographi- cal effect that has to be considered. We have qualitatively addressed this problem by shifting the contact point of the curves by the corresponding height value of the topography image. This allows a direct comparison of force values at given distances from the PL surface plane. This data processing has been already applied to the curves of Fig. 1(b). On top of the PL surface, a long-range attractive force extends to distances as far as $10 \mathrm{~nm}$. On the other hand, the force curve on top of the DNA molecule exhibits a repulsive behavior that extends to similar distances. The EDL nature of the interaction is evident from the exponential dependence of the force. $\lambda_{D}$ values are in agreement with those reported for similar ion concentrations. ${ }^{4}$

The aim of FSI is to represent the images of important sample properties, in this case, the EDL force and perhaps the surface charge density. For that, we need to process the force curves that compose the FSI file. The display of data such as those in Fig. 1(b) for all the points of the map is not an easy task. Even though most commercial AFM setups integrate FSI or similar operation modes, data evaluation has not been standardized. An interesting study is to fit the experimental force curves to some analytic expression. However, due to the complicated geometry of our system, this is out of the scope of the present paper. A fast evaluation of FSI files is to represent isodistance maps. It consists of representing the normal force exerted on the cantilever for a given distance from a base plane. For this purpose, it is necessary to obtain the force versus tip-sample distance representation corrected from height for all the curves as commented above. Figures 1(c) and 1(d) are examples of isodistance maps obtained from the same FSI file as the topography shown in Fig. 1(a), for distances of 3 and $6 \mathrm{~nm}$, respectively. A net repulsive force is clearly observed on top of the molecule for separations from the surface as far as $6 \mathrm{~nm}$, while on top of the PL surface this force is attractive at the same distance. This behavior is homogeneous and reproducible along the whole DNA molecule. It is also remarkable how the resolution of the isodistance maps is improved at lower tipsample distances, which agrees with the exponential decay of the force law. Linear regions of the chain are also clearly resolved.

Another easy way to visualize FSI data is to represent a FSI profile, ${ }^{6,16}$ i.e., a set of force curves recorded while the AFM tip was scanned laterally across a region where a DNA molecule is adsorbed to PL (Fig. 2). In the profile, the three curves in the middle coincide with the positions on top of the DNA strand reaching the maximum repulsive behavior in the middle of the strand. The attractive to repulsive change in the EDL force is also clearly observed,

Equation (1) is usually employed to analyze double layer measurements by AFM. In our case, however, the presence of the DNA molecule has to be taken into account. It represents a surface inhomogeneity, both in topography and surface charge, smaller than the tip. In spite of that, it is interesting to observe that the force dependence remains exponential, with an attractive behavior on top of the PL surface and repulsive on top of the DNA. From Eq. (1) this effect cannot be attributed to a modification in tip-sample distance or in the decay length, as this would only influence the magnitude of the attractive force. The repulsive behavior has to be attributed to the negative surface charge of DNA, although further theoretical studies are needed to extract quantitative values. 


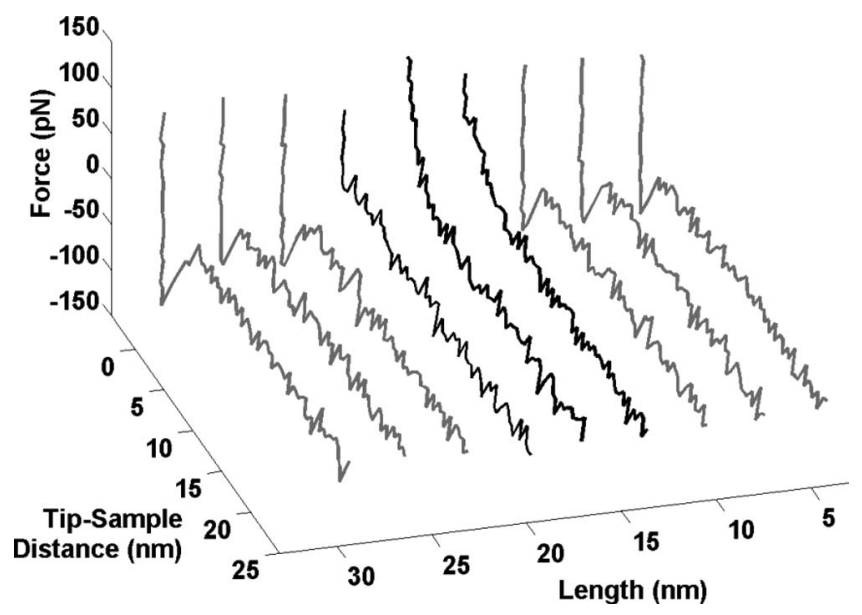

FIG. 2. Successive force scans along a line perpendicular to a DNA strand. Force scans have been extracted from a FSI file transformed into tip-sample representation and the contact point corrected by the corresponding height value. The three middle curves correspond to lateral locations of the DNA strand. Notice that the attractive to repulsive change in the EDL force is strongly dependent on DNA position.

In summary, we have shown that, by operating the AFM in the FSI mode, DNA molecules are resolved in EDL force images.

The authors acknowledge Dr. Sacha Gómez Moñivas for helpful discussions. Financial support has been provided by
Ministerio de Educación y Ciencia through Project Nos. NAN2004-09183-C10-01 and BIO2006-09178-C02-02. J.S. is indebted to CSIC for an I3P predoctoral fellowship.

${ }^{1}$ A. Engel and D. J. Müller, Nat. Struct. Biol. 16, 715 (2000).

${ }^{2}$ N. A. Burnham, R. J. Colton, and H. M. Pollok, Nanotechnology 4, 64 (1993).

${ }^{3} \mathrm{~J}$. N. Israelachvili, Intermolecular and Surface Forces, 2nd ed. (Academic, London, 1992).

${ }^{4}$ H. J. Butt, Biophys. J. 60, 777 (1991).

${ }^{5}$ D. R. Baselt and J. D. Baldeschwieler, J. Appl. Phys. 76, 34 (1994).

${ }^{6}$ M. Radmacher, J. P. Cleveland, M. Fritz, H. G. Hansma, and P. K. Hansma, Biophys. J. 66, 2159 (1994).

${ }^{7}$ K. O. van der Werf, C. A. J. Putman, B. G. de Grooth, and J. Greve, Appl. Phys. Lett. 65, 1195 (1994).

${ }^{8}$ O. H. Willemsen, M. M. E. Snel, K. O. Van der Werf, B. G. de Grooth, J. Greve, P. Hinterdorfer, H. J. Gruber, H. Schindler, Y. Van Kooyk, and C. G. Figdor, Biophys. J. 75, 2220 (1998).

${ }^{9}$ C. Rotsch and M. Radmacher, Langmuir 13, 2825 (1997).

${ }^{10}$ W. F. Heinz and J. H. Hoh, Biophys. J. 76, 528 (1999).

${ }^{11}$ P. Taboada-Serrano, V. Vithayaveroj, S. Yiacoumi, and C. Tsouris, Environ. Sci. Technol. 39, 6352 (2005).

${ }^{12}$ I. Horcas, R. Fernández, J. M. Gómez-Rodríguez, J. Colchero, J. GómezHerrero, and A. M. Baró, Rev. Sci. Instrum. 78, 013705 (2007).

${ }^{13}$ F. Moreno-Herrero, J. Colchero, and A. M. Baró, Ultramicroscopy 96, 16 (2003).

${ }^{14}$ M. Bussiek, N. Mucke, and J. Langowski, Nucleic Acids Res. 31, e137 (2003).

${ }^{15}$ H. J. Butt, B. Cappella, and M. Kappl, Surf. Sci. Rep. 59, 1 (2005).

${ }^{16}$ Y. Martin, C. C. Williams, and H. K. Wickramasinghe, J. Appl. Phys. 61, 4723 (1987). 